\title{
A 40 Month Double-Blind, Randomized, Placebo- Controlled Trial on the Effect of BCc1 Nanomedicine on Survival of Metastatic and Non-Metastatic Gastric Cancer Patients
}

\section{Maryam Hafizi}

Sodour Ahrar Shargh Company

Somayeh Kalanaky

Sodour Ahrar Shargh Company

Hassan Moaiery

Shahid Beheshti University of Medical Sciences

\section{Sajad Noorian}

Sodour Ahrar Shargh Comany

Maryam Khayamzadeh

Shahid Beheshti University of Medical Sciences School

Vahid Kaveh

Iran University of Medical Sciences

\section{Behrooz Gharib}

Naft Hospital

\section{Hossein Fudazi}

Fayazbakhsh Hospital

\section{Mohsen Razavi}

Iran University of Medical Sciences

\section{Arash Jenabian}

Islamic Azad University

\section{Saeid Salimi}

Iran University of Medical Sciences

Mohammad Mahdi Adib Sereshki

Iran University of Medical Sciences

Saideh Fakharzadeh

Sodour Ahrar Shargh Company

Hamid Reza Mirzaee

Shahid Beheshti University of Medical Sciences

Mohammad Esmaeil Akbari

Shahid Beheshti University of Medical Sciences School 
Mohammad Hassan Nazaran ( $\nabla$ mnazaran@nanochelatingtechnology.com )

Sodour Ahrar Shargh Company

\section{Research}

Keywords: BCc1 nanomedicine, Gastric cancer, Nanomedicine, Nanochelating technology, Overall Survival

Posted Date: January 21st, 2021

DOI: https://doi.org/10.21203/rs.3.rs-147862/v1

License: (c) (1) This work is licensed under a Creative Commons Attribution 4.0 International License. Read Full License 


\section{Abstract}

Background: Complementary experiments on nanomedicines as proper candidates for the control and treatment of cancer are widely being conducted nowadays. In the previous study, the effect of BCc1 nanomedicine, which is synthetized based on nanochelating technology, on overall survival (OS) and quality of life of gastric cancer patients was evaluated after 18 months of consumption. The OS of the same patients is reported in this study after 40 months.

Methods: A double-blind, randomized, placebo-controlled, parallel, multicenter design was used in this study. 123 metastatic and non-metastatic gastric cancer patients of both genders (between 25 and 85 years old) participated in this experiment to evaluate their OS after consuming BCc1 for 40 months and to identify the adverse events of this nanomedicine.

Results: The median OS of metastatic patients was 257 days in the BCc1 group [95\% confidence interval (Cl): 144. 142-369.858], while it was 161 days in the placebo [95\% Cl: 118.462-203.538]; hazard ratio $(H R): 0.802$ [95\% Cl: 0.483-1.333] (P-Value $=0.395)$. Similarly, the median OS of non-metastatic patients was 718 days in the $\mathrm{BCc} 1$ group [95\% Cl; 577.706-860.213], while it was 520 days in the placebo [95\% $\mathrm{Cl}$ : 460.280- 580.690]; HR: 0.807 [95\% Cl: 0.343,1.902] (P-Value = 0.624). There was no evidence of adverse events after 40 months.

Conclusion: The OS improvement of metastatic and non-metastatic gastric cancer patients in the previous (18 months of follow-up) and current (40 months of follow-up) studies showed that BCc1 can be used along with base treatments to improve cancer patients' OS.

Trial registration: IRCTID, IRCT2017101935423N1.

Registered on 19 October 2017, http://www.irct.ir/ IRCT2017101935423N1.

\section{Background}

Recent progress in the fields of materials and nanotechnology has resulted in significant improvements in the design of nanomedicines[1]. Nanomedicines are now considered significantly helpful in the successful delivery of therapeutic chemicals to tumor sites in cancer patients[2-4]. The current advances in nanomedicines have led to their broader application in clinical trials. For instance, Abraxane ${ }^{\circledR}$ (paclitaxel)[5] is validated by the US Food \& Drug Administration (FDA) as a useful albumin-bound nanoparticle (NP) for metastatic breast cancer patients. Doxil (doxorubicin) is another NP-based medicine that has been used in multiple-myeloma and ovarian cancer trials and is approved by the FDA[3, $6]$.

The high incidence and yet relatively poor prognosis of gastric cancer as one of the most prevalent types of cancer have encouraged researchers to find a more efficient clinical strategy to manage this diease[7]. Chemotherapy is nowadays considered one common option for the treatment of gastric cancer. However, 
the drug resistance and side effects of such therapy are two major factors limiting the therapeutic efficacy of this kind of treatment[8]. As a result of this, anticancer drug-loaded NPs have been largely used for cancer treatment and have become a key research area in gastric cancer therapy[9-11]. Thus, many laboratory-scale experiments are deemed essential to unlock the full potentiality of these NPs for the treatment of gastric cancer[12].

Nanochelating technology has introduced a new synthesis method in chemistry and nanotechnology[13]. The special nanostructures produced by this technology have been recently utilized at plant[14], animal[15] and human [16]levels. Redistribution of key metal/non-metal elements in cell metabolic cycles, on the basis of the type of disorder, is one of the reasons behind the efficacy of these nanostructures.

As an example, considering the role of magnesium in Alzheimer's disease (AD), Karimi-Sales et al. demonstrated that ALZc3 nanomedicine, synthesized based on nanochelating technology, had better therapeutic effects than memantine and could significantly prevent memory impairment in beta amyloidinduced rat model of $A D[17]$. Moreover, in view of the beneficial impact of zinc and selenium supplementation on diabetes, Fakharzadeh et al. reported that DIBc, a nanochelating-based nano metalorganic framework, had antidiabetic effects on high-fat diet and streptozotocin-induced diabetic rats[18].

In the previous studies, due to the role of iron in the metabolism of cancer cells, BCc1 nanomedicine with iron chelating property was synthesized based on nanochelating technology. This nanomedicine has particles of approximately 30-50 nanometers. Unlike other cancer nanomedicines, BCc1 does not need any carriers and can act independently as a nanomedicine with therapeutic effects[19].

The in vitro study of $\mathrm{BCc} 1$ revealed that this nanomedicine could induce apoptosis in cancer cells. In the animal models, it was shown that BCc1 could decelerate the growth rate of tumors in cancer mice, while improving survival. Moreover, the clinical trial of the 18-month consumption of $\mathrm{BCc} 1$ showed that the median OS of both metastatic and non-metastatic gastric cancer patients who received BCc1 capsules was longer than those who received placebo[3]. In addition, the quality of life (QOL) analysis of the metastatic and non-metastatic gastric cancer patients showed improvements in the BCc1 groups, but the figures in the placebo groups revealed a decrease in scores[3].

Given the positive effects of BCc1 nanomedicine on the OS \& QOL of metastatic and non-metastatic gastric cancer patients after an 18-month follow-up, the impact of this nanomedicine on the OS of the same patients was followed up in the present study after 40 months of consumption.

\section{Methods}

\section{Trial Design and Patients' Eligibility}

The present study was a double-blind, randomized, placebo-controlled, parallel, multicenter clinical trial designed to assess the impact of BCc1 nanomedicine on the OS of metastatic and non-metastatic gastric 
cancer patients as compared to the placebo group. Intervention was added to the patients' base treatment without any interference with the physician's protocol.

The patients were selected from 6 different oncology centers in Tehran, Iran (Shohadaye Tajrish, Bu Ali, Naft Company, Imam Reza, Firouzgar, and Shahid Fayaz Bakhsh hospitals). The patients, clinician, analyzer and researcher in charge were all blinded to the treatment assignment, except for Tehran Darou Pharmaceutical which prepared both $\mathrm{BC} 1$ and placebo capsules for the patients in this project.

The detailed information of the trial was thoroughly explained to the patients by the researcher in charge first, and then the patients' written informed consent to participate in the experiment was received. Yet, they were all allowed to retreat from the experiment at any time without any obligation to provide the reason.

\section{Inclusion / Exclusion Criteria}

The male and female patients who were 25-28 years of age were considered eligible if they were suffering from locally advanced unresectable gastric cancer or were metastatic / non-metastatic adenocarcinoma gastric cancer patients confirmed by their medical records, including endoscopic ultrasound (EUS) and computed tomography scan (CT scan). The grouping classification (c-stage \& ypStage) was performed according to the eighth edition of the American Joint Committee on Cancer (AJCC) staging system guideline. All the patients were capable of swallowing the capsules. Those who declined to take the capsules for one week or showed unacceptable toxicity signs after consumption were excluded from the experiment.

\section{Intervention}

BCc1 nanomedicine was synthesized in the laboratory of Sodour Ahrar Shargh Company based on "Chelate Compounds" technology described in the previous study. BCc1 nanomedicine active pharmaceutical ingredient (API) was put into the same capsules and blister packs as the placebo ones by Tehran Darou Pharmaceutical. This company delivered both the BCc1 and placebo capsules to the researcher in charge, while they were labeled as " $a$ " and " $b$ ' without specifying the content.

The maximum and minimum effective dose of BCc1 nanomedicine were obtained according to LD50 report[3] and in line with the guideline of "Guidance for Industry Estimating the Maximum Safe Starting Dose in Initial Clinical Trials for Therapeutics in Adult Healthy Volunteers" provided and explained in the previous study.

The metastatic gastric cancer patients were administered a daily high dose of $3000 \mathrm{mg} \mathrm{BCc} 1$ nanomedicine, while non-metastatic patients received a daily low dose of $1500 \mathrm{mg} \mathrm{BCc1}$ nanomedicine. They continued to receive the treatment until they passed away or when a decision was made to stop the treatment. At first, both the BCc1 and placebo capsules were given to the patients by the researcher in 
charge to be consumed for one month. Then, after making sure the patients had taken the drugs, the researcher in charge provided them with sufficient capsules again for the following month. Both the BCc1 and placebo capsules were administered free of charge.

\section{Endpoint}

The patients' OS was the endpoint of the present study. It was defined as the period from the patients' randomization until their death, irrespective of the cause.

\section{Adverse Events}

The same adverse events that were mentioned in the previous study, including vomit, nausea, neutropenia and anorexia as well as vital signs, were followed up in the present study after 40 months of consumption. These indices were checked every other month. Hematological and biochemical blood tests were conducted every six months. National Cancer Institute Common Toxicity Criteria (version 4.0) was used to assess the adverse events of BCc1 nanomedicine.

\section{Sample Size \& Grouping}

148 gastric cancer patients were checked for eligibility on the basis of the aforementioned criteria, and 123 of them were selected and assigned to two main groups of metastatic (63 patients) and nonmetastatic (60 patients) according to "Consolidated Standards of Reporting Trials[20] (Fig.1, 2 ). Then, the patients in each main group were randomly placed in the BCc1 and placebo groups according to block randomization form, regardless of the physician's desire. After the analysis of the study results and the patients' documents, it was revealed that there were three similar types of regimens (FOLFOX, DCF and FLOT) used along with BCc1 nanomedicine or placebo for some of the patients.

\section{Data Collection}

The patients' information, consistency and missing values were collected and checked by the researcher in charge. Comprehensive data management details are available upon request.

\section{Statistical Methods}

In this study, the Kaplan-Meier and Lifetime Table was used to assess the survival distribution of the patients in both the BCc1 and placebo groups. The log rank test was also used to test whether there was a significant difference between the OS of the groups. Using Cox regression model, the hazard ratios (HRs) and their 95\% confidence interval were obtained[21]. To test the hypothesis, a convectional 0.05 
significant level was adopted. All statistical data analyses were performed using SPSS software (version 25; SPSS Inc., Chicago, IL, USA)[22].

\section{Results}

\section{Patients' Disposition and Characteristics}

The patients were recruited between Oct. 26, 2016 and Feb. 6, 2020. Their characteristics (supplementary data 1,2), both metastatic and non-metastatic, are thoroughly explained in the previous report of this clinical study[3].

\section{Median OS of Metastatic and Non-Metastatic Gastric Cancer Patients}

As can be seen in Table 1, the median OS of the metastatic patients in the BCc1 and placebo groups was 257 [95\% Cl:144.142-369.858] and 161 days [95\% Cl:118.462-203.538], respectively. The results showed that the median OS of the metastatic patients who received BCc1 nanomedicine was 96 days more than that of the placebo patients; HR: 0.802 [95\% Cl: 0.483-1.333]; $p=0.39$. The Kaplan-Meier diagram of survival and hazard function diagram of the metastatic patients are presented in Fig. 3A, B. 
Table 1

Median Overall Survival Analysis of Metastatic and Non-Metastatic Gastric Cancer Patients

Group

Metastatic

Non-Metastatic

Total Number

$\mathrm{BCc} 1$

Placebo BCc1

Placebo

718

520

Median (Day)

257

161

57.581

$21.703 \quad 72.068$

30.717

Std. Error

144.142

118.462

577.706

460.280

Median 95\% Cl (Lower)

369.858

$203.538 \quad 860.213$

580.690

Median 95\% Cl (Upper)

P-Value for Testing Equality of Survival $\quad 0.392$

Distributions

0.624

0.802

0.807

HR

[95\% Cl: $(0.483,1.333)$

[95\% Cl: $(0.343,1.902)$;

(BCc1/Placebo)

P-value $=0.395]$

P-value $=0.624]$

[95\% Cl for HR;

\section{P-value]}

Equality Assay of Survival Distributions (for BCc1 and Placebo Groups) by Log Rank (Mantel-Cox)

Similarly, the median OS of the non-metastatic patients in the BCc1 and placebo groups was 718 [95\% Cl: 577.706-860.213] and 520 days [95\% Cl:460.280-580.690], respectively. According to the results, the 
median OS of the non-metastatic patients who received BCc1 nanomedicine was 198 days more than that of the placebo patients; HR: 0.807 [95\% Cl: 0.343-1.902; $p=0.62$. The Kaplan-Meier diagram of survival and hazard function diagram of the non-metastatic patients are presented in Fig. 4A, B.

\section{Median OS of Patients Receiving BCc1 or Placebo Without Chemotherapy}

After analyzing the data, it was revealed that some of the patients received only either the BCc1 or placebo capsules without any other treatments during the experiment. The analysis result of these subgroups was as follows:

As can be seen in Table 2, the median OS of the metastatic patients who received BCc1 or placebo without chemotherapy was 257 days [95\% Cl: 78.175-435.825] in the BCc1 group and 129 days [95\% Cl: 76.132-181.868] in the placebo. As the results show, the median OS of the metastatic patients who received only $\mathrm{BCc} 1$ nanomedicine was 128 days more than that of those who received only placebo; HR: 0.641 [95\% Cl: $0.285-1.446] ; p=0.280$. The Kaplan-Meier diagram of survival and hazard function diagram of the metastatic patients who received only the intervention (BCc1 nanomedicine or placebo) are shown in Fig. 5A, B. 
Table 2

Median Overall Survival Analysis of the Patients Who Only Received Intervention (BCc1 nanomedicine or Placebo Without Chemotherapy)

\begin{tabular}{|c|c|c|c|c|}
\hline \multicolumn{5}{|l|}{ Group } \\
\hline & \multicolumn{2}{|c|}{ Metastatic } & \multicolumn{2}{|c|}{ Non-Metastatic } \\
\hline Total Number & BCc1 & Placebo & BCc1 & Placebo \\
\hline & 257 & 129 & 863.313 & 519.868 \\
\hline \multicolumn{5}{|l|}{ Median (Day) } \\
\hline Std. Error & 91.237 & 26.974 & 102.264 & 32.345 \\
\hline Median 95\% Cl (Lower) & 78.175 & 76.132 & 662.876 & 456.471 \\
\hline Median 95\% Cl (Upper) & 435.825 & 181.868 & 1063.751 & 583.265 \\
\hline \multicolumn{5}{|c|}{$\begin{array}{l}\text { P-Value for Testing Equality of Survival } \\
\text { Distributions }\end{array}$} \\
\hline & \multicolumn{2}{|l|}{0.280} & \multicolumn{2}{|l|}{0.281} \\
\hline HR & \multicolumn{2}{|l|}{0.641} & \multicolumn{2}{|l|}{0.426} \\
\hline (BCc1/Placebo) & \multicolumn{2}{|c|}{ [95\% Cl: $(0.285,1.446)$} & \multicolumn{2}{|c|}{ [95\% Cl: $(0.086,2.111)$} \\
\hline [95\% Cl for HR; & \multicolumn{2}{|c|}{$P$-value $=0.284]$} & \multicolumn{2}{|c|}{ P-value $=0.296]$} \\
\hline \multicolumn{5}{|l|}{ P-value] } \\
\hline Equality Assay of Surviv & $\mathrm{Cc} 1$ and $\mathrm{Pl}$ & Groups) by & Log Rank ( & -Cox) \\
\hline
\end{tabular}

Similarly, the median OS of the non-metastatic patients who received BCc1 or placebo without chemotherapy was 863 days [95\% Cl: 662.876-1063.751] in the BCc1 group and 519 days [95\% Cl: 465.471-583.265] in the placebo. Accordingly, the median OS of the non-metastatic patients who received only BCc1 nanomedicine was 344 days more than the median OS of those who received only placebo; HR: 0.426 [95\% Cl: 0.086-2.111]; $p=0.281$. The Kaplan-Meier diagram of survival and hazard function diagram of the non-metastatic patients who received only the intervention (BCc1 nanomedicine or placebo) are presented in Fig. 6 A, B.

\section{Death Percentage Analysis}


As shown in Table 3A, 91.2\% of the metastatic patients in the BCc1 group and $93.5 \%$ of the metastatic patients in the placebo group passed away during the 40-month experiment. In non-metastatic patients, the death percentages were $29 \%$ and $34 \%$ in the BCc1 and placebo groups, respectively.

Table 3

A) Death Percentage Analysis of Metastatic and Non-Metastatic Gastric Cancer Patients

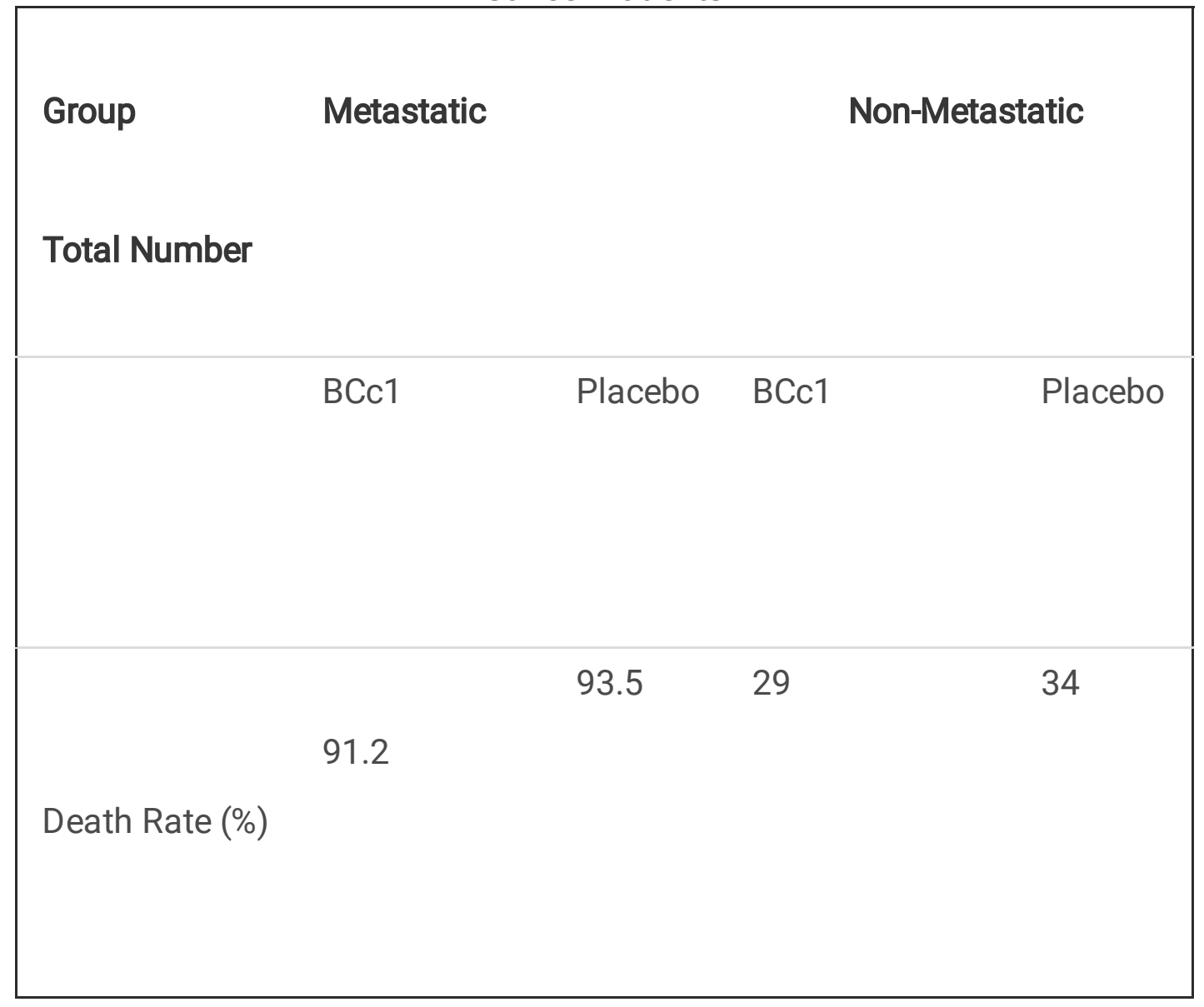

Table 3

B) Death Percentage Analysis of Metastatic and Non-Metastatic Gastric Cancer Patients (Without Chemotherapy)
Group
Metastatic
Non-Metastatic

Total Number

BCc1

Placebo BCc1

Placebo

Death Rate (\%) 94

91

16

38 
In addition, as shown in Table 3B, 94\% of the metastatic patients who received only BCc1 nanomedicine without chemotherapy and $91 \%$ of the metastatic patients who received only placebo without chemotherapy passed away during the 40-mount experiment. In non-metastatic patients, the death percentages were $16 \%$ and $38 \%$ for those who received only BCc1 or placebo, respectively.

\section{Adverse Events}

The adverse events of the patients were followed up according to CTCAE 4.0. No adverse $[23,24]$ events were reported during the 40-month follow-up of the patients as shown in Table 4. The results of the patients' whole blood test are reported in Table 5, and the renal and hepatic functions are shown in Tables $6 \mathrm{~A}$, and $6 \mathrm{~B}$, respectively.

Table 4

Adverse Events Fallow-Up According to CTCAE 4.0.

\begin{tabular}{|ll|}
\hline Adverse Events & Follow-Up Results after 40 Months \\
\hline Hand-Foot Syndrome & Not \\
\hline Pain & Not \\
\hline Neutropenia & Not \\
\hline Bilirubin Increased & Not \\
\hline Fatigue & Not \\
\hline Mucositis & Not \\
\hline Thrombocytopenia & Not \\
\hline Hematuria & Not \\
\hline Anorexia & Not \\
\hline Dizziness & Not \\
\hline Fever & Not \\
\hline Diarrhea & Not \\
\hline Skin Ulceration & Not \\
\hline Vomiting & Not \\
\hline Dyspnea & Not \\
\hline
\end{tabular}


Table 5

Whole Blood Function

\begin{tabular}{|lll|}
\hline INDEX & UNIT & AMOUNT \\
\hline WBC & $x 1000 / m m 3$ & $5.77 \pm 1.5$ \\
\hline RBC & Mill $/ \mathrm{mm} 3$ & $4.35 \pm 0.4$ \\
\hline HGB & $\mathrm{g} / \mathrm{dl}$ & $13.07 \pm 0.8$ \\
\hline HCT & $\%$ & $38.65 \pm 4.0$ \\
\hline MCV & $\mathrm{FI}$ & $91.32 \pm 8.1$ \\
\hline MCH & $\mathrm{pg}$ & $30.85 \pm 2.3$ \\
\hline MCHC & $\mathrm{g} / \mathrm{dl}$ & $33.77 \pm 1.1$ \\
\hline Platelet & $\mathrm{x} 1000 / \mathrm{mm} 3$ & $219 \pm 11$ \\
\hline RDW & $\%$ & $34.8 \pm 18.1$ \\
\hline PDW & $\mathrm{Fl}$ & $13.8 \pm 0.5$ \\
\hline MPV & $\mathrm{FI}$ & $9.2 \pm 1.8$ \\
\hline
\end{tabular}

Table 6

A) Renal Function

\begin{tabular}{|lll|}
\hline INDEX & UNIT & AMOUNT \\
\hline Urea & $\mathrm{mg} / \mathrm{dl}$ & $30.1 \pm 11.6$ \\
\hline Creatinine & $\mathrm{mg} / \mathrm{dl}$ & $1.03 \pm 0.04$ \\
\hline
\end{tabular}


Table 6

B) Hepatic Function

\begin{tabular}{|lll|}
\hline INDEX & UNIT & AMOUNT \\
\hline Triglycerides & $\mathrm{mg} / \mathrm{dl}$ & $85.5 \pm 48$ \\
\hline Total Cholesterol & $\mathrm{mg} / \mathrm{dl}$ & $157 \pm 19$ \\
\hline AST (SGOT) & $\mathrm{U} / \mathrm{L}$ & $21.85 \pm 3.1$ \\
\hline ALT (SGPT) & $\mathrm{U} / \mathrm{L}$ & $13.62 \pm 3.2$ \\
\hline Alkaline Phosphatase & $\mathrm{IU} / \mathrm{L}$ & $185 \pm 100$ \\
\hline Bilirubin (Total) & $\mathrm{mg} / \mathrm{dl}$ & $0.95 \pm 0.08$ \\
\hline Bilirubin (Direct) & $\mathrm{mg} / \mathrm{dl}$ & $0.36 \pm 0.1$ \\
\hline
\end{tabular}

\section{Discussion}

Although chemotherapy is considered a helpful cancer therapy, in most cases, cancer patients become resistant to chemotherapy medicines during the treatment, and therefore normal cancer cases progress to advanced stages[25]. According to the American Society of Clinical Oncology (ASCO), the 5-year survival rate of the gastric cancer patients who are diagnosed and treated before the spread of the cancer outside stomach is $69 \%$. The percentage is $31 \%$ if the cancer spreads to the surrounding tissues or organs and/or the regional lymph nodes, and finally the rate reduces to $5 \%$ if it spreads to distant parts in the body[26] .

Improvements in the field of nanotechnology have provided a more potential strategy for resolving the above-mentioned problem and its negative consequences. Greater penetration into tissues at molecular level, improved drug localization and cellular uptake, higher feasibility to program NPs to identify cancer cells, healthy cells interaction avoidance and larger surface area are some of the properties of nanomedicines that are helpful in controlling and treating cancers[27]. Resolving the challenges of synthesizing controllable, reproducible and scalable NPs as well as improving NPs screening and evaluation will facilitate clinical development[28-30].

Nanochelating technology is a branch of nanotechnology and has recently succeeded in introducing novel nanomedicines that do not need any carriers as their new synthesis method can improve their performance. The anticancer effect of one of these nanomedicines on gastric cancer patients was evaluated in the present study.

Numerous studies have demonstrated the important role of iron in inducing oxidative damage in cancer initiation and development[31, 32]. It is well-known that neoplastic cells express high levels of transferrin 
receptor 1 and that cancer cells are highly in need of iron. It is also well-proven that iron metabolism is the downside of all kinds of neoplastic cells, and iron maldistribution in cancer patients[33,34] often causes the disturbance of many physiological processes such as hemoglobin synthesis[35], and as a result of this, anemia is detected in approximately $40 \%-70 \%$ of all cancer patients[36, 37]. Therefore, in view of the vital role of iron in cell physiological[38] processes as well as hematopoiesis, the need for an agent that can redistribute iron well without excreting it seems essential, otherwise the anemia could be exacerbated.

In dozens of studies, the antineoplastic effects of iron chelators are evaluated in vitro, in vivo and in clinical trials. In cell culture as well as animal studies, the efficacy of several iron chelators has been already proven and the mechanisms of their antineoplastic activity (inducing cell cycle arrest, inhibition of ribonucleotide reductase, up-regulation of Ndrg1 and increase in P53 expression) are determined.

Desferrioxamine (DFO) is an FDA-approved iron chelator as the recommended first-line therapy for iron overload in patients with thalassemia major[39]. The anticancer effects of DFO are also widely evaluated in cell culture and animal models[40,41], but due to its poor oral bioavailability and short plasma half-life, it must be administered via slow subcutaneous administration during 8-12 hours, 5-7 days/week, often resulting in poor compliance[42].

Triapine is another iron chelator that is widely evaluated in clinical trials for different neoplastic diseases, revealing that its antineoplastic effect is due to the combined action of iron chelation and free radicals generation[43-45]. A number of conducted studies on Triapine have failed to meet the minimal efficacy, while several studies have demonstrated the opposite. It should be noted that the consumption of this iron chelator in these studies caused several adverse effects such as methemoglobinemia, fatigue, nausea and vomiting.

Therefore, according to the proven role of iron in cancer initiation, development and progress, and in view of the above-mentioned studies, it seems essential to use an efficient technology to design a safer and more efficient agent that has iron chelating property and is capable of the smart management of iron redistribution without inducing the adverse effects of iron excretion, such as anemia.

In the previous study, it was revealed that BCc1 nanomedicine has iron chelating and antineoplastic properties. This nanomedicine could induce apoptosis and cell arrest in cancer cells as well as their genes expression[19]. In a cancer animal model, it was demonstrated that the mixture of BCc1 nanomedicine and doxorubicin could increase the mean OS of the cancer mice by $48 \%$, where doxorubicin dose was reduced by 50 times[46].

In a clinical trial, the effect of BCc1 nanomedicine on metastatic and non-metastatic gastric cancer patients was evaluated for the first time. The follow-up results of consuming BCc1 after 18 and 40 months showed that the median OS of the metastatic patients who received BCc1 was 112 and 96 days more than those who took placebo, respectively. Likewise, the median OS of the non-metastatic patients in the BCc1 group was 184 and 198 days more than those in the placebo group[3]. 
Moreover, the analysis of the patients showed that consuming this nanomedicine, despite having iron chelating property, had no negative impact on cell blood count, ferritin, transferrin iron binding capacity and other performance characteristics of iron $[3,19]$. In addition, the evaluation of a number of liver function-related characteristics and enzymes showed their normal performance in the BCc1 groups.

As a result, considering the iron chelating property of $\mathrm{Bcc} 1$ nanomedicine without its negative impact on important iron-related properties in the body as well as the positive impact of this nanomedicine on the OS of cancer patients, it could be claimed that BCc1 nanomedicine can inhibit cancer development by intelligently affecting iron metabolism without any adverse effect on physiological indices.

Furthermore, in the previous study, the analysis of the median OS of the patients who only used BCc1 or placebo showed that the median differences were in favor of the BCc1 groups with 121 and 12-day differences in the metastatic and non-metastatic groups, respectively. The same analysis in the present study showed that the differences increased from 121 to 128 days in the metastatic patients and from 12 to 30 days in the non-metastatic patients. These results indicate that $\mathrm{BCc} 1$ nanomedicine could be regarded as an independent anticancer nanomedicine deserving further investigations in the future.

Since the design of small molecules capable of targeting several pathways at the same time is a serious challenge in the field of cancer therapy, discovering the precise impacting mechanisms of BCc1 nanomedicine novel structure through further scientific and experimental researches can introduce it as an invaluable nanomedicine for controlling and treating cancer cells.

\section{Conclusions}

The results indicate that not only does $\mathrm{BCc} 1$ nanomedicine have an iron-chelating property and prevent cancer cells accessibility to iron, but it also benefits from the unique properties of nanostructures. One major reason for such effect is the intelligent redistribution of vital elements in cell metabolic cycle according to the type of disorder. This redistribution is the result of a novel form of chelated nanostructures that are produced by nanochelating technology.

\section{Abbreviations}

Food and Drug Administration (FDA); Overall Survival (OS); Quality of Life (QOL); Endoscopic ultrasound (EUS); Computed Tomography scan (CT scan); American Joint Committee on Cancer staging system guideline (AJCC); Active Pharmaceutical Ingredient (API); Hazard ratios (HRs); Alzheimer's disease (AD) ; Confidence Interval (Cl); useful albumin-bound nanoparticle (NP) ; quality of life (QOL) ; Desferrioxamine (DFO) ;American Society of Clinical Oncology (ASCO).

\section{Declarations}

\section{Ethics Approval and Consent to Participate}


This trial is registered with Iranian Registry of Clinical Trials; Reg. No. IRCT2017101935423N1. It has also received the ethics approval of Shahid Beheshti University of Medical Sciences, Tehran, Iran; Reg. No. IR.SBMU.REC.1396.2.

\section{Consent for Publication}

Not applicable

\section{Availability of Data Materials}

The datasets generated during the current study are available from the corresponding author upon reasonable request.

\section{Funding}

Not applicable

\section{Competing Interests}

The authors declare that they have no competing interests.

\section{Authors' Contributions}

$\mathrm{MH}$ conducted most of the experiments, collected the data and wrote the manuscript. SK performed the synthesis of $\mathrm{BCC} 1$ nanomedicine. SN and MKH carried out the statistical analyses.

VK, BG, HF, MR, AJ, SS, MA, and HM collected the data and recruited the patients. MEA designed the study. MHN contributed to the conception and design of BCc1 nanomedicine. All of the authors have read and approved the final manuscript.

\section{Acknowledgements}

The Department of Research and Development at Sodour Ahrar Shargh Company and Cancer Research Centre at Shahid Beheshti University of Medical Sciences supported this work. We also appreciate the contribution of Dr Mosavi and Dr Abdolkarimi (the CEO and member of directors' board at Tehran Darou Pharmaceutical Co., Tehran, Iran) to this study.

\section{References}


1. Gu, W., et al., Actively targeted nanomedicines for precision cancer therapy: Concept, construction, challenges and clinical translation. J Control Release, 2020.

2. Singh, M., et al., Application of gold nanoparticles for gastrointestinal cancer theranostics: $A$ systematic review. Nanomedicine, 2015. 11(8): p. 2083-98.

3. Hafizi, M., et al., A randomized, double-blind, placebo-controlled investigation of BCc1 nanomedicine effect on survival and quality of life in metastatic and non-metastatic gastric cancer patients. $J$ Nanobiotechnology, 2019. 17(1): p. 52.

4. Paradise, J., Regulating Nanomedicine at the Food and Drug Administration. AMA J Ethics, 2019. 21(4): p. E347-355.

5. Amiri-Kordestani, L., et al., A Food and Drug Administration analysis of survival outcomes comparing the Adjuvant Paclitaxel and Trastuzumab trial with an external control from historical clinical trials. Ann Oncol, 2020.

6. Barenholz, Y., Doxil(R)-the first FDA-approved nano-drug: lessons learned. J Control Release, 2012. 160(2): p. 117-34.

7. Bray, F., et al., Global cancer statistics 2018: GLOBOCAN estimates of incidence and mortality worldwide for 36 cancers in 185 countries. CA Cancer J Clin, 2018. 68(6): p. 394-424.

8. Beeharry, M.K., et al., Optimization of perioperative approaches for advanced and late stages of gastric cancer: clinical proposal based on literature evidence, personal experience, and ongoing trials and research. World J Surg Oncol, 2020. 18(1): p. 51.

9. Cunningham, D., et al., Capecitabine and oxaliplatin for advanced esophagogastric cancer. $\mathrm{N}$ Engl J Med, 2008. 358(1): p. 36-46.

10. Yang, F., et al., Gastric cancer combination therapy: synthesis of a hyaluronic acid and cisplatin containing lipid prodrug coloaded with sorafenib in a nanoparticulate system to exhibit enhanced anticancer efficacy and reduced toxicity. Drug Des Devel Ther, 2018. 12: p. 3321-3333.

11. Namiki, Y., et al., Nanomedicine for cancer: lipid-based nanostructures for drug delivery and monitoring. Acc Chem Res, 2011. 44(10): p. 1080-93.

12. Guerrero, A.R., et al., Gastric Cancer: Nanoparticles as Tools to Improve Treatment Efficacy. Curr Pharm Des, 2016. 22(39): p. 5988-5997.

13. MH., N., Chelate compounds.Google Patents US8288587B2. 2012.

14. Fakharzadeh, S., et al., Using Nanochelating Technology for Biofortification and Yield Increase in Rice. Sci Rep, 2020. 10(1): p. 4351.

15. Hossein Ali Ghasemi, I.H., Maryam Hafizi, Kamran Taherpour, Mohammad Hassan Nazaran, Effect of advanced chelate technology based trace minerals on growth performance, mineral digestibility, tibia characteristics, and antioxidant status in broiler chickens. Nutr Metab (Lond), 2020.

16. Hafizi, M., et al., GFC7 as a Smart Growth Nanofactor for ex vivo Expansion and Cryoprotection of Humans' Hematopoietic Stem Cells. Int J Nanomedicine, 2020. 15: p. 6263-6277. 
17. Karimi-Sales, R., et al., Neuroprotective Effect of New Nanochelating-Based Nano Complex, ALZc3, Against Abeta (1-42)-Induced Toxicity in Rat: a Comparison with Memantine. Pharm Res, 2020. 37(3): p. 48.

18. Fakharzadeh, S., et al., DIBC nano metal-organic framework improves biochemical and pathological parameters of experimental chronic kidney disease. J Trace Elem Med Biol, 2020. 61: p. 126547.

19. Kalanaky, S., et al., BCC1, the novel antineoplastic nanocomplex, showed potent anticancer effects in vitro and in vivo. Drug Des Devel Ther, 2016. 10: p. 59-70.

20. Fiteni, F., et al., Endpoints in cancer clinical trials. J Visc Surg, 2014. 151(1): p. 17-22.

21. Langman, M.J., et al., Prospective, double-blind, placebo-controlled randomized trial of cimetidine in gastric cancer. British Stomach Cancer Group. Br J Cancer, 1999. 81(8): p. 1356-62.

22. Quintero, D.e.a., Workload Optimized Systems: Tuning POWER7 for Analytics.

23. https://ctep.cancer.gov/protocoldevelopment/electronic_applications/ctc.htm.

24. https://www.eortc.be/services/doc/ctc/ctcae_4.03_2010-06-14_quickreference_5x7.pdf.

25. Housman, G., et al., Drug resistance in cancer: an overview. Cancers (Basel), 2014. 6(3): p. 1769-92.

26. Cancer.Net, S.C.S.

27. Farjadian, F., et al., Nanopharmaceuticals and nanomedicines currently on the market: challenges and opportunities. Nanomedicine (Lond), 2019. 14(1): p. 93-126.

28. Shi, J., et al., Cancer nanomedicine: progress, challenges and opportunities. Nat Rev Cancer, 2017. 17(1): p. 20-37.

29. Akcan, R., et al., Nanotoxicity; a challenge for future medicine. Turk J Med Sci, 2020.

30. Riehemann, K., et al., Nanomedicine--challenge and perspectives. Angew Chem Int Ed Engl, 2009. 48(5): p. 872-97.

31. Chang, V.C., M. Cotterchio, and E. Khoo, Iron intake, body iron status, and risk of breast cancer: a systematic review and meta-analysis. BMC Cancer, 2019. 19(1): p. 543.

32. Zhou, L., et al., Alterations in Cellular Iron Metabolism Provide More Therapeutic Opportunities for Cancer. Int J Mol Sci, 2018. 19(5).

33. Petzer, V., I. Theurl, and G. Weiss, Established and Emerging Concepts to Treat Imbalances of Iron Homeostasis in Inflammatory Diseases. Pharmaceuticals (Basel), 2018. 11(4).

34. Wang, Y., et al., Iron Metabolism in Cancer. Int J Mol Sci, 2018. 20(1).

35. Ganz, T. and E. Nemeth, Iron sequestration and anemia of inflammation. Semin Hematol, 2009. 46(4): p. 387-93.

36. Ludwig, $\mathrm{H}$., et al., Iron metabolism and iron supplementation in cancer patients. Wien Klin Wochenschr, 2015. 127(23-24): p. 907-19.

37. Hung, N., et al., Risk of cancer in patients with iron deficiency anemia: a nationwide population-based study. PLoS One, 2015. 10(3): p. e0119647. 
38. Fonseca-Nunes, A., et al., Body iron status and gastric cancer risk in the EURGAST study. Int J Cancer, 2015. 137(12): p. 2904-14.

39. Modell, B., et al., Survival and desferrioxamine in thalassaemia major. Br Med J (Clin Res Ed), 1982. 284(6322): p. 1081-4.

40. de Souza, A.L., et al., Comparing desferrioxamine and light fractionation enhancement of ALA-PpIX photodynamic therapy in skin cancer. Br J Cancer, 2016. 115(7): p. 805-13.

41. Kicic, A., A.C. Chua, and E. Baker, Desferrithiocin is a more potent antineoplastic agent than desferrioxamine. Br J Pharmacol, 2002. 135(6): p. 1393-402.

42. Nisbet-Brown, E., et al., Effectiveness and safety of ICL670 in iron-loaded patients with thalassaemia: a randomised, double-blind, placebo-controlled, dose-escalation trial. Lancet, 2003. 361(9369): p. 1597-602.

43. Chaston, T.B., et al., Examination of the antiproliferative activity of iron chelators: multiple cellular targets and the different mechanism of action of triapine compared with desferrioxamine and the potent pyridoxal isonicotinoyl hydrazone analogue 311. Clin Cancer Res, 2003. 9(1): p. 402-14.

44. Kolesar, J., et al., Population pharmacokinetics of 3-aminopyridine-2-carboxaldehyde thiosemicarbazone (Triapine(R)) in cancer patients. Cancer Chemother Pharmacol, 2011. 67(2): p. 393-400.

45. Kunos, C.A., et al., Phase I trial of daily triapine in combination with cisplatin chemotherapy for advanced-stage malignancies. Cancer Chemother Pharmacol, 2017. 79(1): p. 201-207.

46. Hafizi, M., et al., Effects of $B C C 1$ nanoparticle and its mixture with doxorubicin on survival of murine $4 T 1$ tumor model. Onco Targets Ther, 2019. 12: p. 4691-4701.

\section{Figures}




\section{Enrollment}

Assessed for eligibility ( $n=73)$

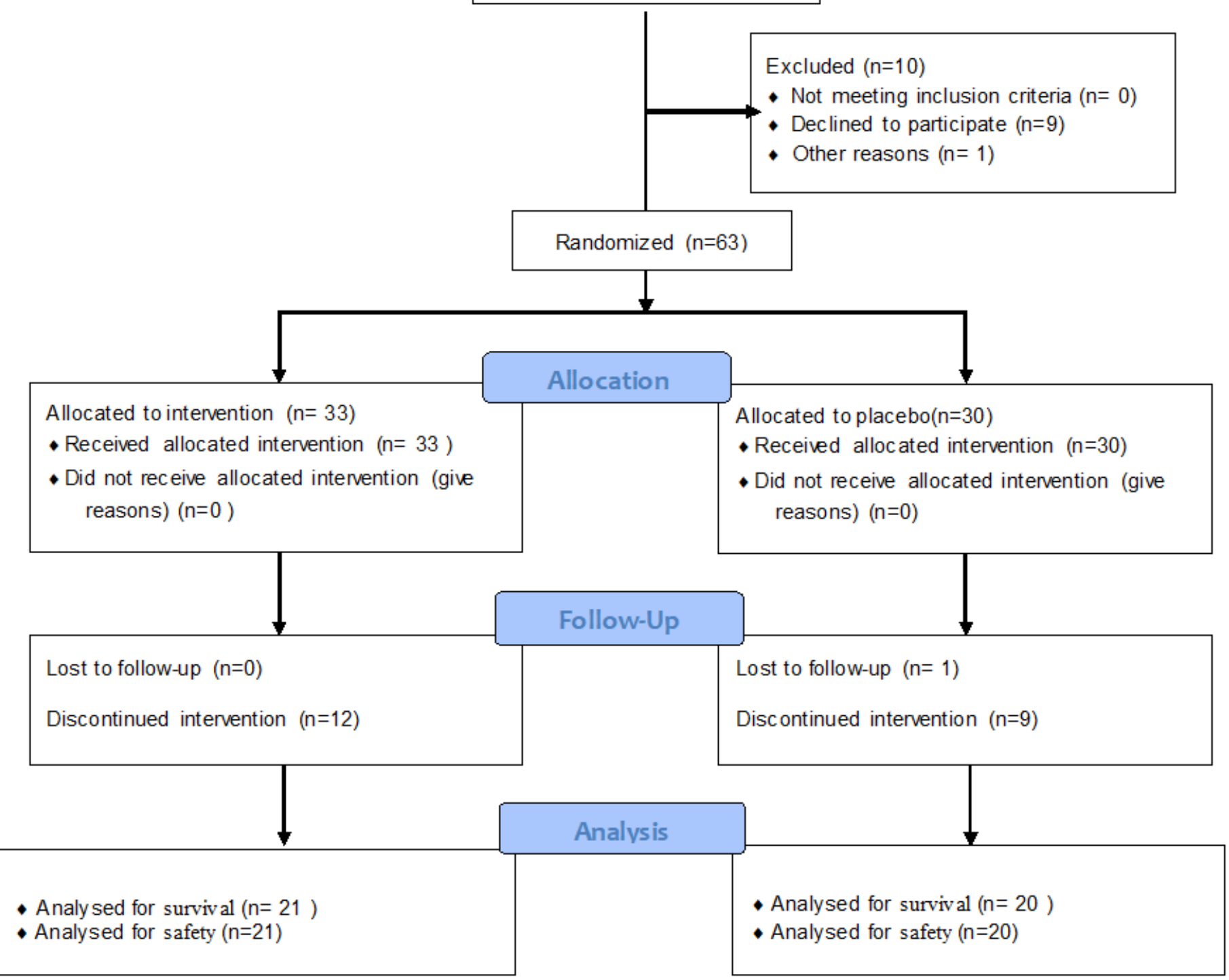

Figure 1

Consort Flow Diagram for patients with metastatic gastric cancer. 


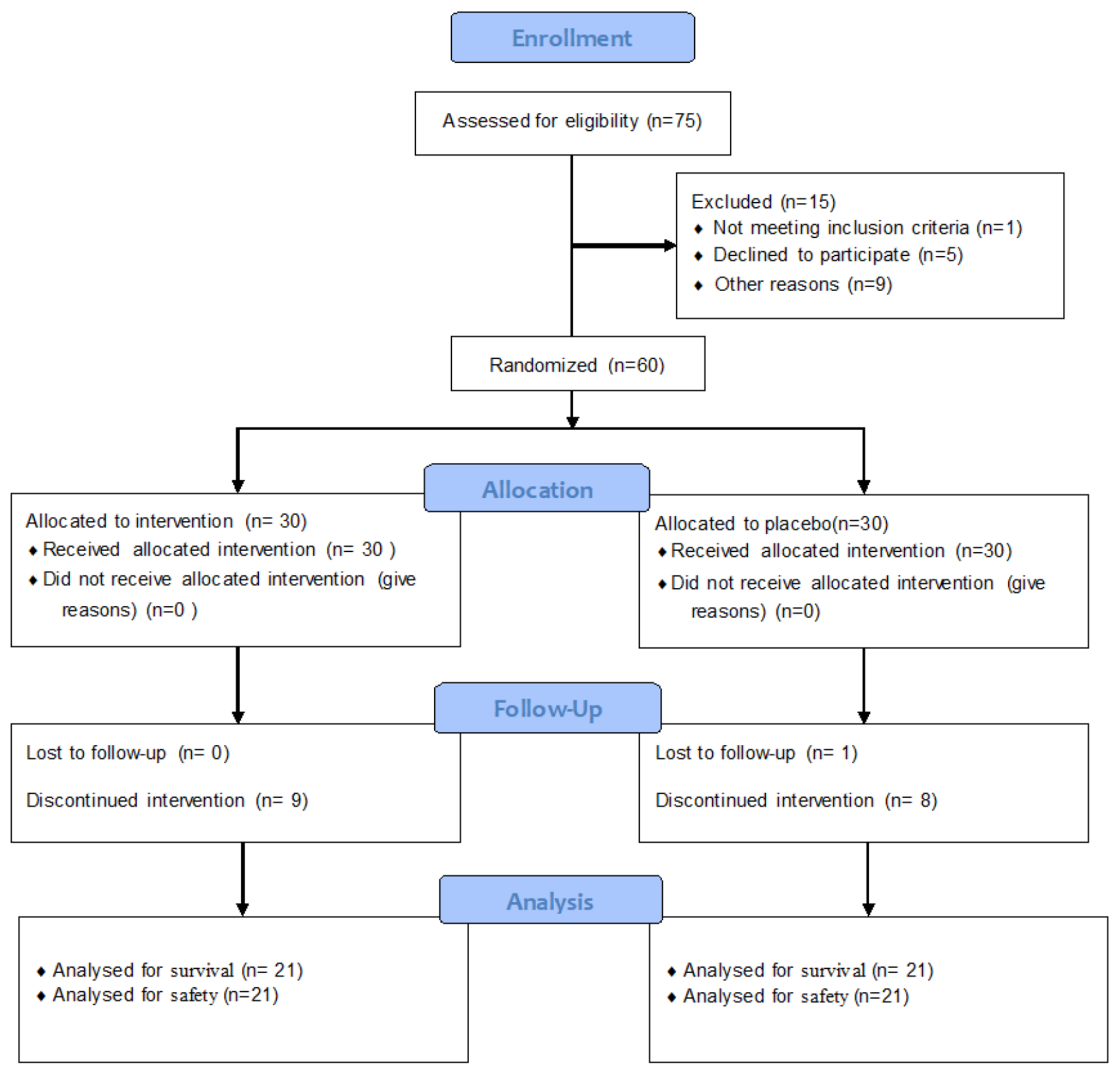

Figure 2

Consort Flow Diagram for patients with non-metastatic gastric cancer. 

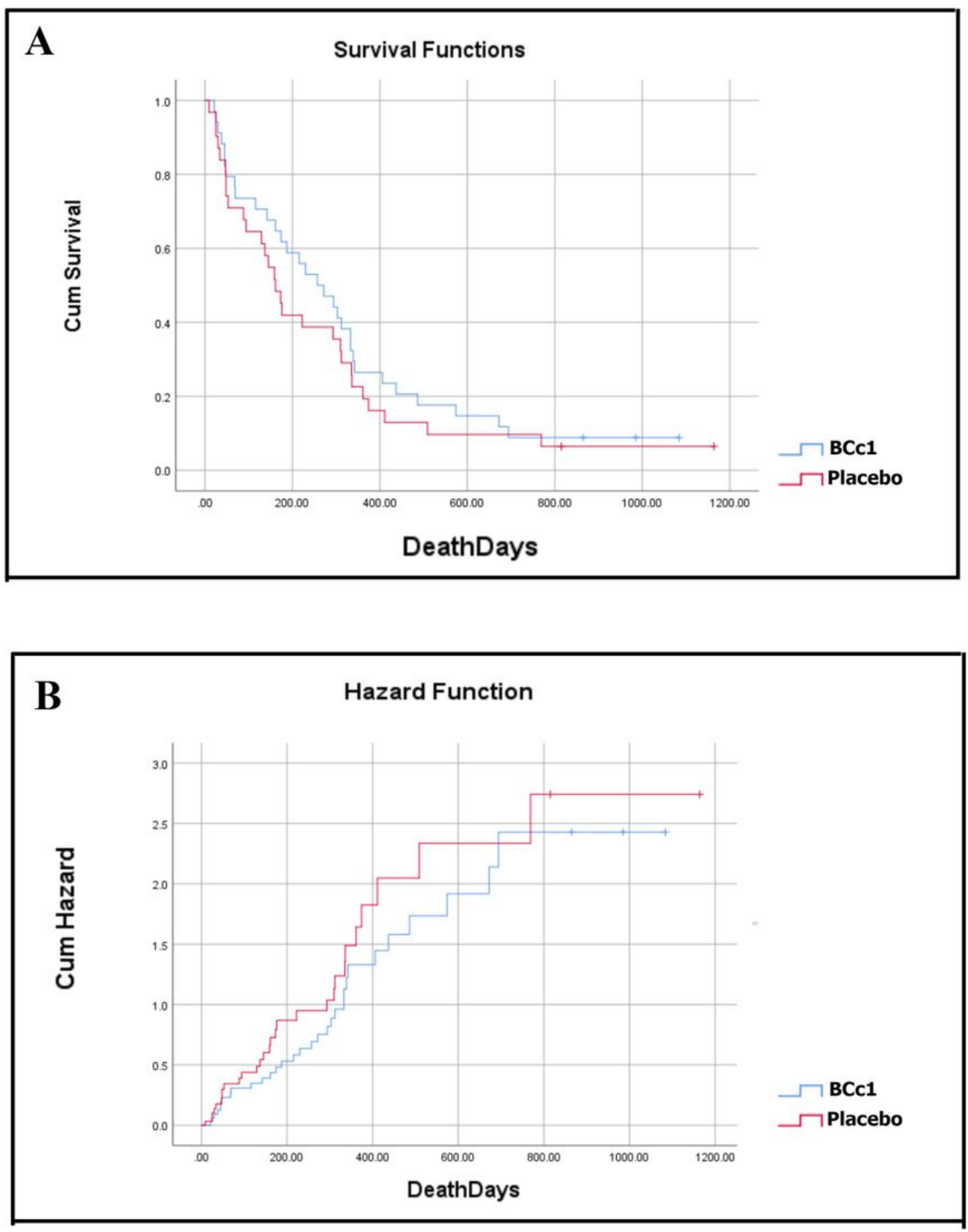

Figure 3

A) Kaplan-Meier diagram of survival B) Hazard function diagram for metastatic patients (Lifetime tables of hazard and survival for patients who received BCc1 nanomedicine show a higher survival and lower hazard than patients who received placebo). 

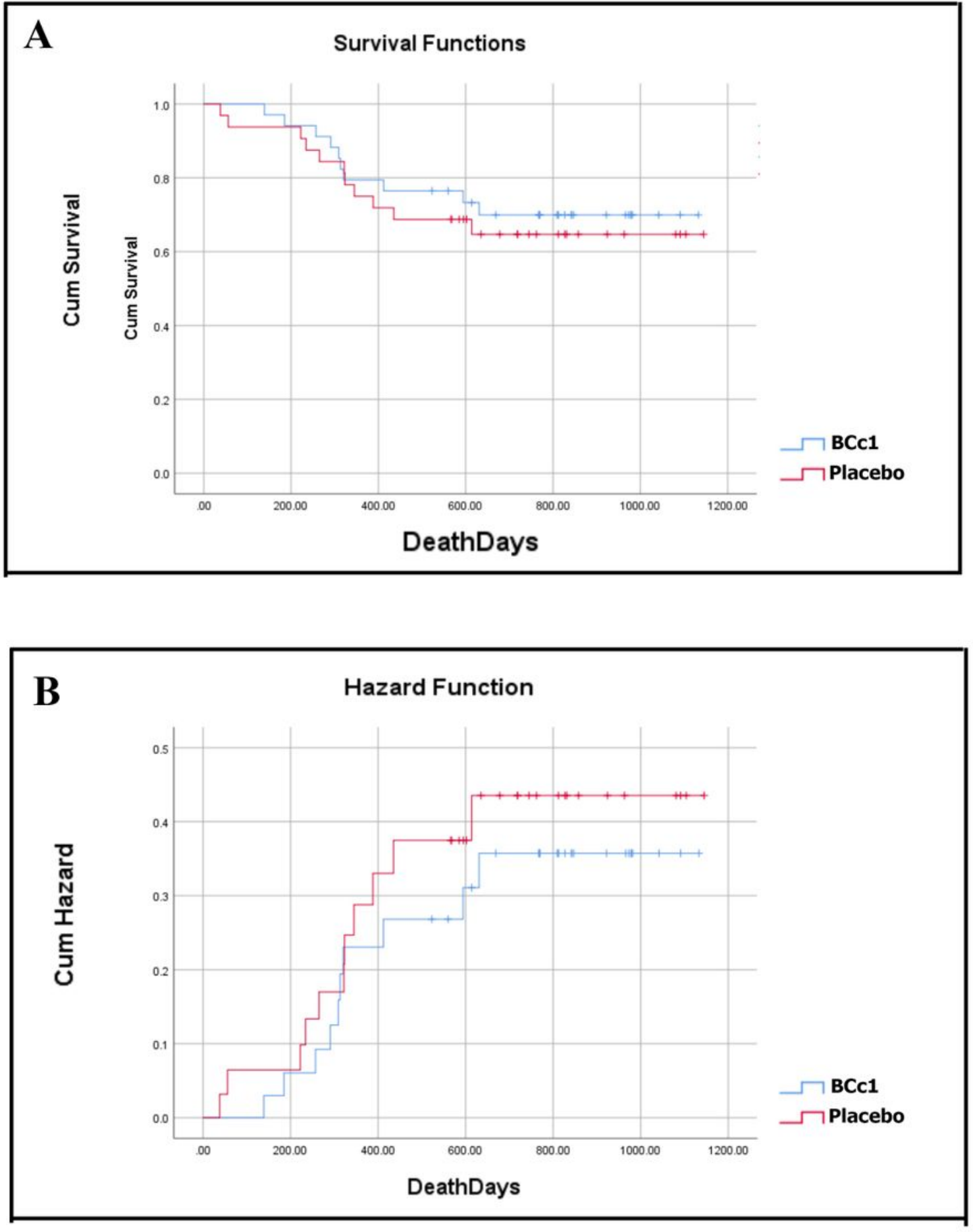

Figure 4

A) Kaplan-Meier diagram of survival B) Hazard function diagram for non-metastatic patients (Lifetime tables of hazard and survival for patients who received BCc1 nanomedicine show a higher survival and lower hazard than patients who received placebo). 

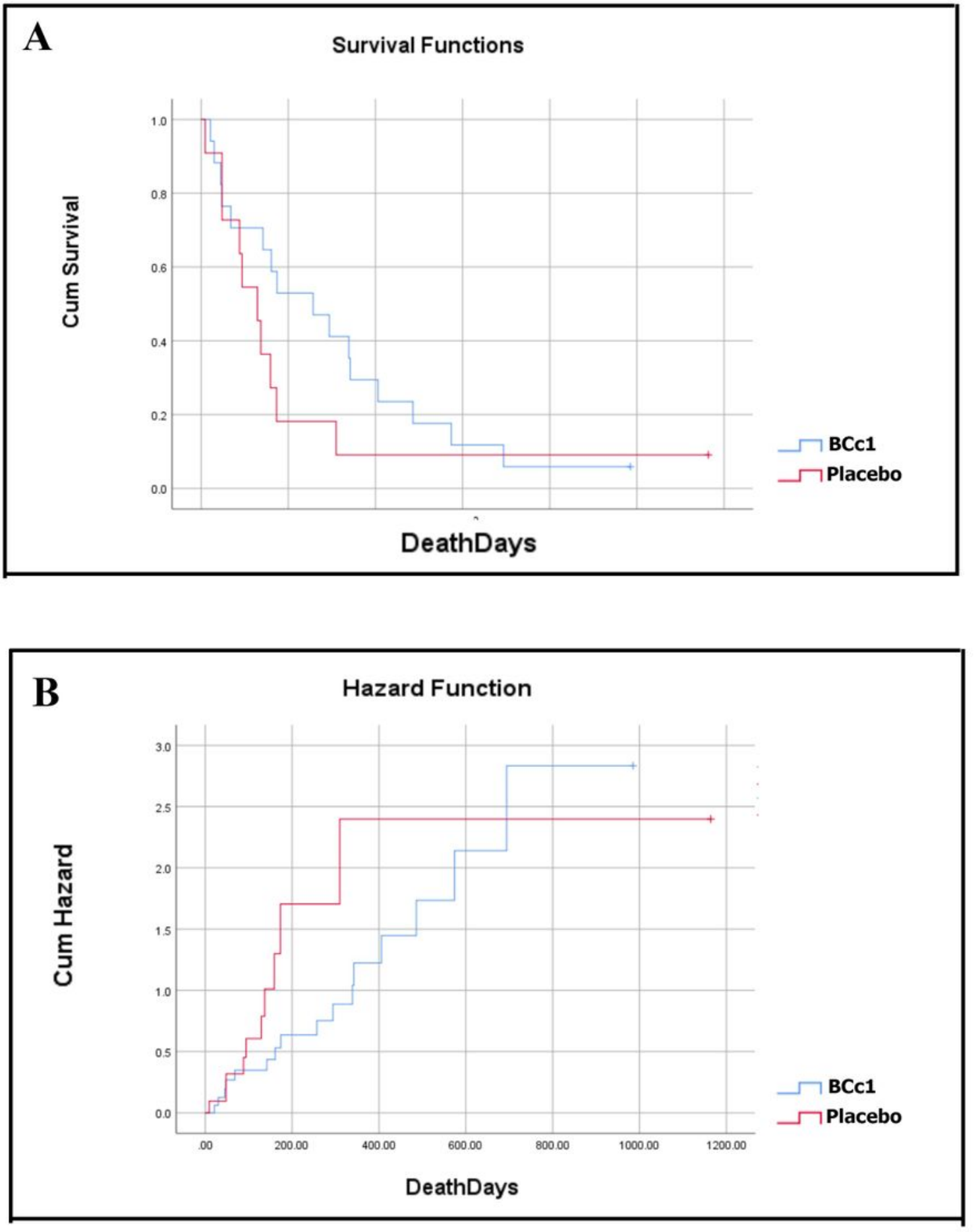

\section{Figure 5}

A) Kaplan-Meier diagram of survival B) Hazard function diagram for metastatic patients who only received interventions (BCc1 nanomedicine or placebo) without undergoing chemotherapy. 

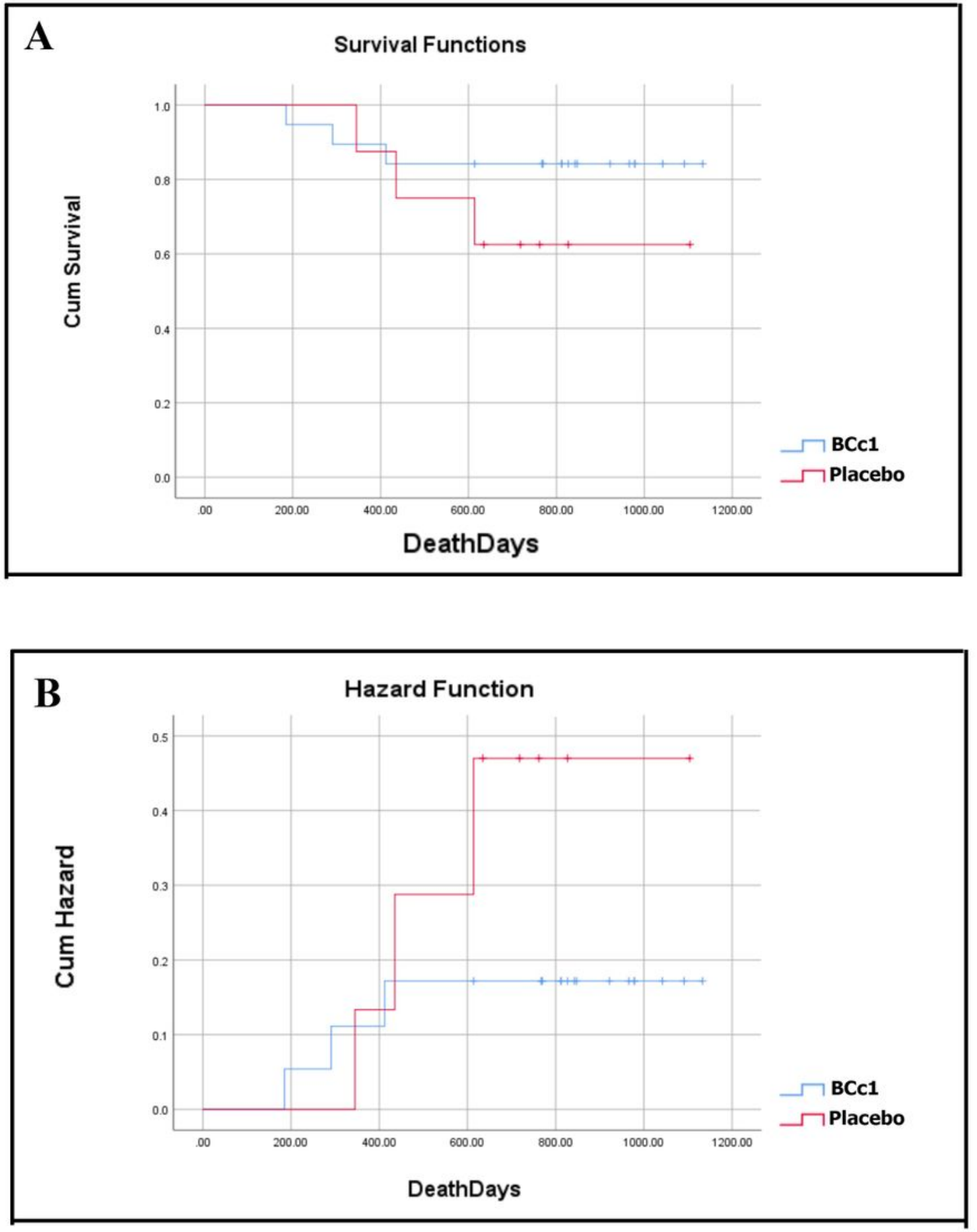

Figure 6

A) Kaplan-Meier diagram of survival B) Hazard function diagram for non-metastatic patients who only received interventions (BCc1nanomedicine or placebo) without undergoing chemotherapy.

\section{Supplementary Files}


This is a list of supplementary files associated with this preprint. Click to download.

- SupplementaryData1.docx

- SupplementaryData2.docx 